\title{
Computed Tomography Findings in Spinal Compression in 196 Dogs
}

\author{
Robert Cristian PURDOIU ${ }^{1 *}$, Reut ASHUR ${ }^{1}$, Laura CONDOR ${ }^{1}$, Radu LĂCĂTUȘ ${ }^{1}$ \\ ${ }^{1}$ Department of Semiology, Ethopathology and Medical imaging. University of Agricultural Sciences and \\ Veterinary Medicine, Cluj Napoca. \\ *corresponding author: robert.purdoiu@usamvcluj.ro
}

Bulletin UASVM Veterinary Medicine 75(1)/2018

Print ISSN 1843-5270; Electronic ISSN 1843-5378

doi:10.15835/buasvmcn-vm:003417

\begin{abstract}
Spinal cord lesion can be presented as acute, in cases of trauma such as fractures, hematomas and inflammation, or can progress slowly in some chronic diseases.

The aim of the study was to identify the sensitivity and specificity of CT examination in diagnostics of different causes of spinal compression in dogs. In this study, different cases with same nature of progressed signs and no findings on x-ray imaging were presented for investigation. All cases were demonstrated for spinal impact sings according to the neurological examination and were good candidates for CT imaging. The study was conducted on 196 dogs, evaluated through CT examination between 2015 and 2017. All dogs arrived with signs suggesting spinal cord lesion of varying degrees, those sings progressed with time but had no evident of spinal lesion in $\mathrm{x}$-ray imaging. Different diagnostics were made based on the CT scans and the cases were divided based on that.

For the dogs in the study, signs of spinal compression were evaluated, being taken in consideration the presence of hypo or hyper attenuating material in the medullary canal, integrity of the spinal canal and the vertebral body, the values of $A$ and $H$ index, location of the spinal lesion and presence of vacuum phenomena.

Computed tomography examination of the vertebral column proved to be a valuable tool that completed the neurologic examination and show a high sensitivity (94.48\%) and a high specificity (87.88\%) in diagnostic of spinal cord compression.
\end{abstract}

Keywords: spinal cord, dog, Computed Tomography, disc protrusion

\section{Introduction}

Spinal cord lesion can be presented as acute impact, in cases of trauma such as: fractures, hematomas and inflammation, or can progress slowly, in a chronic course, in cases of: degenerative disc disease, stenosis of vertebral canal, compression impact by various tumors or bone hypertrophy. The degree of spinal cord compression does not correlate with the severity of the sign, therefore dogs with acute onset of signs may present sever neurological symptoms despite mild compression of the spinal cord (Freeman et al., 2010). One of the most common condition found in dogs is the intervertebral disc degeneration (IVDD), is an unknown etiology condition that produce herniation or extrusion of the intervertebral disc material into the vertebral canal (Hoerlein, 1971; Lim et al., 2010). In case of acute disc protrusions (Hansen type I), studies show that the plain radiography have a relevance of $69-72 \%$ of the cases (Kirberger et al., 1992) but false positive or false negative results are common using this method (Lamb, 1994). Plain radiography is a necessary tool in running out other possible pathology of the spine but is necessary to use other imagistic method to define the landmark of the lesion and confirm the diagnostic (Liptak et a.l, 1999; Olby et al., 2000; Sharp and Wheeler, 2005). Development of technology and scanning procedures made possible a multiplanes imaging evaluation of the spine with a very thin section, CT scanning being used to evaluate the spine junction 
for IVDD and the spinal cord for tumors (Turel et al., 1986; Chambers et al., 1994; Drost et al., 1996).

The aim of the study was to identify the sensitivity and specificity of CT in diagnostics of different causes of spinal compression in dogs and to highlight the changes that occur in that given situation.

\section{Material and method}

Different cases with same nature of progressed signs and no findings on $\mathrm{x}$-ray imaging were presented for investigation. All cases were demonstrated for spinal impact sings according to the neurological examination and were good candidates for CT imaging. Different diagnostics were made based on the CT scans and the cases were divided based on that.

The study was conducted on 196 dogs, evaluated through CT between 2015 and 2017, in the radiology laboratory of the Faculty of Veterinary Medicine Cluj Napoca, Romania. All dogs arrived with signs suggesting spinal cord lesion of varying degrees, the sings progressed with time and at the radiography evaluation there were no evident sign of spinal lesion on x-ray imaging. The patients were clinically and neurologically examined by the doctor that requested the CT evaluation or by the doctor that performed the CT evaluation, depending if the patient was referred or ambulatory. After sedation, the patient was restrained in dorsal decubitus and a CT scan of the spine was performed.

Neurological examination was performed according to basic neurologic test and evaluation methods. The neurological examination was focused on evaluation of spinal reflexes - tendon reflexes (patellar, biceps and triceps), flexion reflexes (thoracic and pelvic limb-withdrawal), perineal (anal), cutaneous trunci (panniculus) and evaluation of nociception (pain sensation) - "superficial" and "deep pain" perception. Each evaluation results were recorded as present, weak or absent.

Evaluation of patient was graded according to modified Tarlov scale for better fitting the patient to this study. The Tarlov scale is a 5-point scale to assess upper and lower limb locomotion and uses scores ranging from 0 (paraplegia) to 4 (normal locomotor function) (Sra et al, 1999).

The patients were classified as grades I to IV. Grade I being the patients that present only mild pain and grade IV being the patients that have loss the limb function and the deep pain.

Scans were performed using a CT scanner (Siemes, Somatomscope model) under general anesthesia. Anesthesia protocol was performed using Diazepam (Gedeon Richter, Romania) $0.25 \mathrm{mg} / \mathrm{kg}$ in combination with Ketamine (Romvac, Romania) $1 \mathrm{mg} / \mathrm{kg}$ and Propophol (APP Pharmaceuticals) to effect, maintenance of anesthesia was done using Propophol to effect. For each patient, a lateral and ventrodorsally topograms were obtained. A helical scanning at $3 \mathrm{~mm}$ slice interval with a mean of $130 \mathrm{kVp}$ and $24 \mathrm{mAs}$ was performed for each patient and the reconstruction was made at $1 \mathrm{~mm}$.

The degree of attenuation of the normal spinal cord was measured in Housefield units (HU) in at least 5 consecutive images, in each dog, that were at least $5 \mathrm{~mm}$. Disc herniation pattern was classified as single, continuous, and multiple. Single pattern was disc herniation located at only one intervertebral disc space. Continuous pattern was disc herniation present at more than two adjacent intervertebral disc spaces. Multiple patterns, described discs herniation present at more than two sites.

The place of herniation was described as either ventral or lateral (left, right). A herniation was regarded as ventral when the apex of the herniated disc material was localized in the ventral region of the spinal cord and as lateral when the apex was close to the lateral recess.

For calculation of relation between herniated disc and the vertebral canal the A-index and $\mathrm{H}$-index were used. The A-index represents the ratio of the area of the herniated disc and the vertebral canal and the $\mathrm{H}$-index is the ration of the height of the herniated disc to the vertebral canal. The formula for A-index calculation is (area of the herniated disc) / (area of the spinal canal) $\times 100$ (\%), and for $\mathrm{H}$-index calculation (the height of herniated disc material / the height of spinal canal) $\times 100(\%)($ Lim et al, 2010). Measurements were obtained in transvers images using HOROS DICOM viewer (figure 1, figure 2). The attenuation of the herniated material was also measured.

Statistical analysis was performed using One Way ANOVA to confirm the correlation of clinical grade with $\mathrm{A}$-index and $\mathrm{H}$-index, and the difference of the attenuation of herniated disc material. 


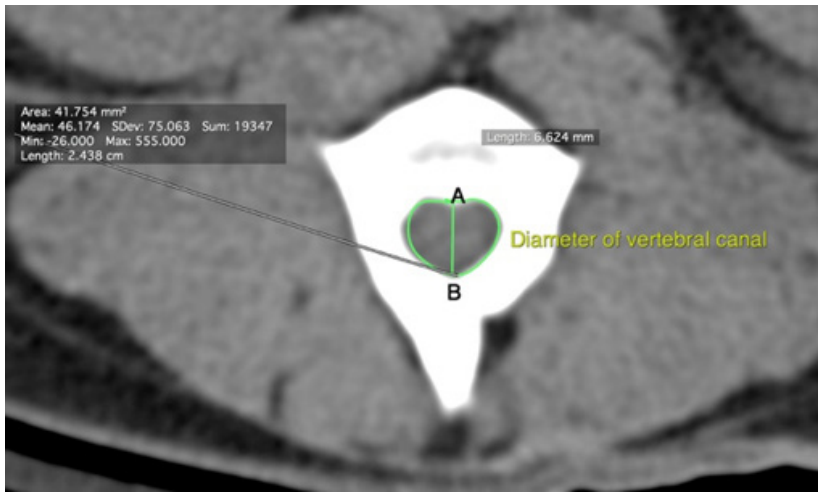

Figure 1. Area of the vertebral canal. The AB line is the height of the vertebral canal

In the normal vertebra, the margin should be smooth with high attenuation value as normal bone. Inflammation of the bone or the surrounding tissue can influence the shape, continuation and attenuation of the bone margin. The vertebral body margins were evaluated based on their surface continuation, the surface was appreciated as continuous, smooth or irregular and the attenuation of the margin was recorded. Narrowing of vertebral body disc space can be an indication for vertebral disc disease, degeneration compression or herniation.

The intervertebral vacuum phenomenon (VP) in the dog describes an accumulation of gas in the crevices of intervertebral discs or adjacent vertebrae (Feng et a.l, 2011; D’Anastasi et al., 2011). Mostly the gas is represented by nitrogen and it occurs primarily after vaporization of solute gases in the extracellular fluids in fissures of degenerative vertebral discs but can also arise following a sudden fall in pressure, for example, after a vertebral disc herniation (Knutson, 1942; Feng et al., 2011; D'Anastasi et al., 2011).

Hyperplastic changes in the spine were evaluated by their location in comparison to the spinal cord. It can be either laterally (left or right), dorsal or ventral.

Hyperplastic lesion was appreciated based on the type of tissue affected, bone destruction if the lesion invaded the vertebra, spinal nerve if the lesion invaded the intervertebral foramen or transverse canal, or mixed, if both area effected. If the lesion has invaded either the vertebral canal or intervertebral space the area affected was measured as the width between 2 bony structures, it has occupied.

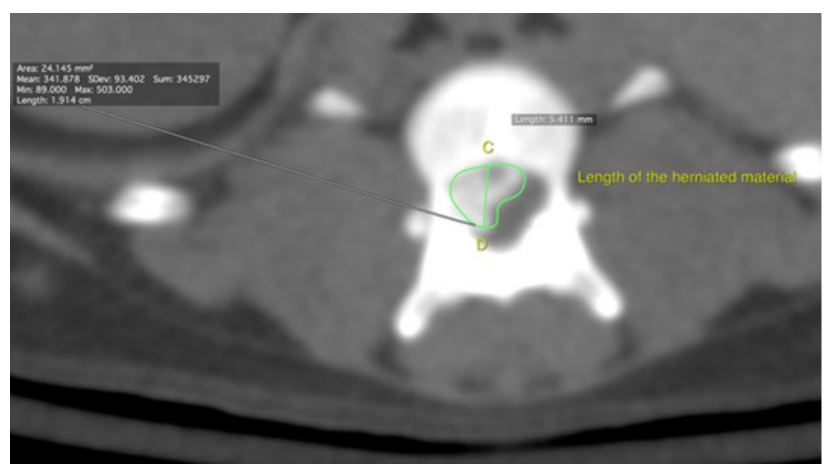

Figure 2. The area and the height (CD line) of the herniated material

In the patients that a disc disease was suspected base on the clinical signs but the CT show no disc material, contrast substance (Omnipaque 350 or Visipaque 320, GE Healthcare) in dose of 2 $\mathrm{ml} / \mathrm{kg}$ IV was administered to identify a possible inflammation that compress the spinal cord. The CT myelography $(0.2 \mathrm{ml} / \mathrm{kg})$ was performed only in one case diagnosed with discospondilitis, for the rest of the cases the assessment of disc protrusion was performed based on the accumulation of contrast media on the inflammation site. The CT evaluation of the spine with contrast was performed after 3 minutes of I.V. contrast bolus injection was done.

\section{Results and discussions}

From the total of 196 cases studied, based on the anamnesis, neurological examination and CT results, 163 cases were diagnosed with intervertebral degenerative disc disease (IVDD). From the cases diagnosed with IVDD ( $N=163)$, a total of 9 cases tested false negative on native CT, in those cases the inflammation produced by the protruded disc was highlighted after I.V. contrast was administered. From the total cases studied 4 cases tested false positive for disc compression, but in those cases the compression was due to tumoral processes, another 29 cases were tested true negative due to inflammatory processes located on the spine (myelitis, lysis of the vertebral body) The cases diagnosed with IVDD ( $\mathrm{N}=163)$ were classified according to breed in large $(\mathrm{N}=27)$, medium large $(\mathrm{N}=31)$, medium small $(\mathrm{N}=61)$ and small breeds $(\mathrm{N}=44)$. From the total cases diagnosed with IVDD, large breeds represented $16 \%(\mathrm{n}=27)$, medium large breeds $19 \%(\mathrm{~N}=31)$, 
Table 1. Breed distribution of cases

\begin{tabular}{cccccccc}
\hline Large breed & \multicolumn{2}{c}{ Medium large breeds } & \multicolumn{2}{c}{ Medium small breeds } & \multicolumn{2}{c}{ Small breeds } \\
\hline $\begin{array}{c}\text { Germen Shepherd } \\
\text { Central Asia } \\
\text { Shepherd }\end{array}$ & 6 & Malinois & 5 & Caniche & 24 & $\begin{array}{c}\text { Yorkshire } \\
\text { Terrier }\end{array}$ & 23 \\
\hline $\begin{array}{c}\text { Golden Retriever } \\
\text { Caucazian }\end{array}$ & 6 & Mixed breed & 10 & $\begin{array}{c}\text { French } \\
\text { buldog }\end{array}$ & 14 & Teckel & 8 \\
\hline Shepherd & 2 & & Mixed breed & 6 & Mixed breed & 4 \\
\hline Labrador & 5 & & & & & & Pechingese \\
\hline
\end{tabular}

Table 2. Disc pattern herniation according to breed

\begin{tabular}{ccccc}
\hline \multirow{2}{*}{ Pattern } & \multicolumn{4}{c}{ Breeds } \\
\cline { 2 - 5 } & Large & Medium-Large & Medium-Small & Small \\
\hline Single & 23 & 26 & 45 & 29 \\
\hline Multiple & 3 & 5 & 14 & 13 \\
\hline Continuous & 1 & 0 & 2 & 2 \\
\hline
\end{tabular}

Table 3. Affected intervertebral spaces

\begin{tabular}{cccccccccccccc}
\hline \multicolumn{3}{c}{ Cer. Vert. } & \multicolumn{4}{c}{ Thoracic vertebra } & \multicolumn{3}{c}{ Lumbar vertebra } & \multicolumn{2}{c}{ Sacrum } \\
\hline Cases & $2-3$ & $9-10$ & $10-11$ & $11-12$ & $12-13$ & $13-1$ & $1-2$ & $2-3$ & $3-4$ & $4-5$ & $5-6$ & $6-7$ & $7-1$ \\
\hline
\end{tabular}

Table 4. Disc pattern herniation of the affected intervertebral space

\begin{tabular}{|c|c|c|c|c|c|c|c|c|c|c|c|c|c|c|}
\hline & \multirow{2}{*}{$\frac{\text { Cer. Vert. }}{2-3}$} & \multicolumn{4}{|c|}{ Thoracic vertebra } & \multirow[b]{2}{*}{$13-1$} & \multicolumn{6}{|c|}{ Lumbar vertebra } & \multicolumn{2}{|c|}{ Sacrum } \\
\hline & & $9-10$ & $10-11$ & $11-12$ & $12-13$ & & $1-2$ & $2-3$ & $3-4$ & $4-5$ & $5-6$ & $6-7$ & $7-1$ & 1 \\
\hline Single & 2 & 1 & 6 & 9 & 18 & 23 & 6 & 11 & 17 & 4 & 7 & 4 & 11 & 0 \\
\hline Multiple & 0 & 0 & 0 & 5 & 9 & 8 & 3 & 3 & 4 & 3 & 1 & 1 & 0 & 0 \\
\hline Continuous & 0 & 0 & 0 & 0 & 2 & 3 & 1 & 0 & 0 & 0 & 0 & 1 & 0 & $\overline{0}$ \\
\hline
\end{tabular}

Table 5. Locations of all affected disc spaces

\begin{tabular}{|c|c|c|c|c|c|c|c|c|c|c|c|c|c|c|}
\hline & \multirow{2}{*}{$\frac{\text { Cer. Vert. }}{2-3}$} & \multicolumn{5}{|c|}{ Thoracic vertebra } & \multicolumn{6}{|c|}{ Lumbar vertebra } & \multicolumn{2}{|c|}{ Sacrum } \\
\hline & & $9-10$ & $10-11$ & $11-12$ & $12-13$ & $13-1$ & $1-2$ & $2-3$ & $3-4$ & $4-5$ & $5-6$ & $6-7$ & $7-1$ & 1 \\
\hline Ventral & 2 & 1 & 4 & 8 & 17 & 27 & 9 & 10 & 15 & 4 & 7 & 2 & 11 & 0 \\
\hline Left Lat & 0 & 0 & 2 & 4 & 5 & 5 & 1 & 4 & 2 & 0 & 1 & 2 & 0 & 0 \\
\hline Right Lat & 0 & 0 & 0 & 2 & 7 & 2 & 0 & 0 & 4 & 3 & 0 & 2 & 0 & 0 \\
\hline
\end{tabular}

medium small breeds $37 \%(\mathrm{~N}=61)$ and the small breeds represented $27 \%(\mathrm{~N}=44)$ (table 1).

The disc protrusions were recorded according to breed and herniated pattern (table 2), according to the intervertebral spaces involved (table 3), according the herniated pattern and intervertebral disc (table 4) and according to the position of the protruded material in the vertebral canal (table 5) (figure 3 , figure 4 ).
Base on the Tarlov scale the cases were split in groups as follow: Grade I - 24 cases, Grade II - 63 cases, Grade III - 52 cases, Grade IV - 24 cases.

Attenuation of the normal spine, according to a similar study conducted by Lim in 2010, was 23.4 $\pm 11.8 \mathrm{HU}$ with a range of -0.6 to $59.3 \mathrm{HU}$. In our study, the normal values of the spinal cord were $29.2 \pm 14.9 \mathrm{HU}$ with a range of -7 to $70 \mathrm{HU}$.

The ANOVA test show a correlation between the case grade and A-index ( $<<0.05)$, for the Grade 


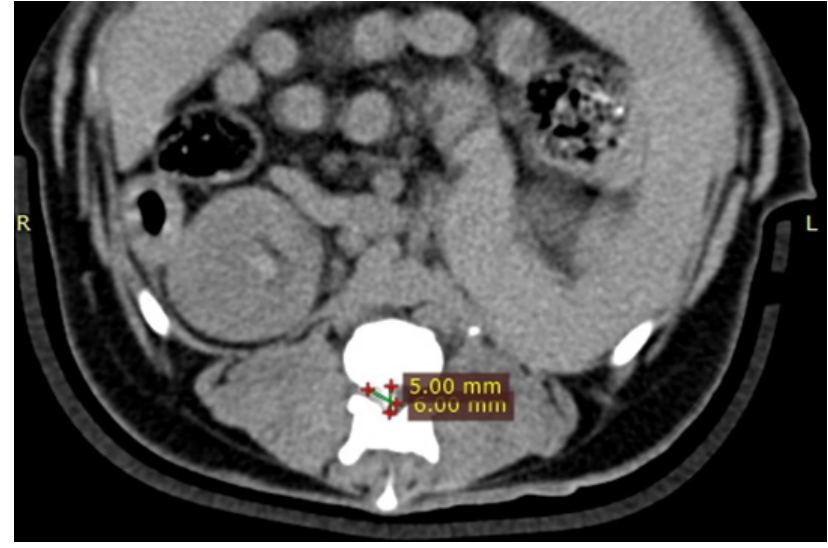

Figure 3. Lateral discal protrusion

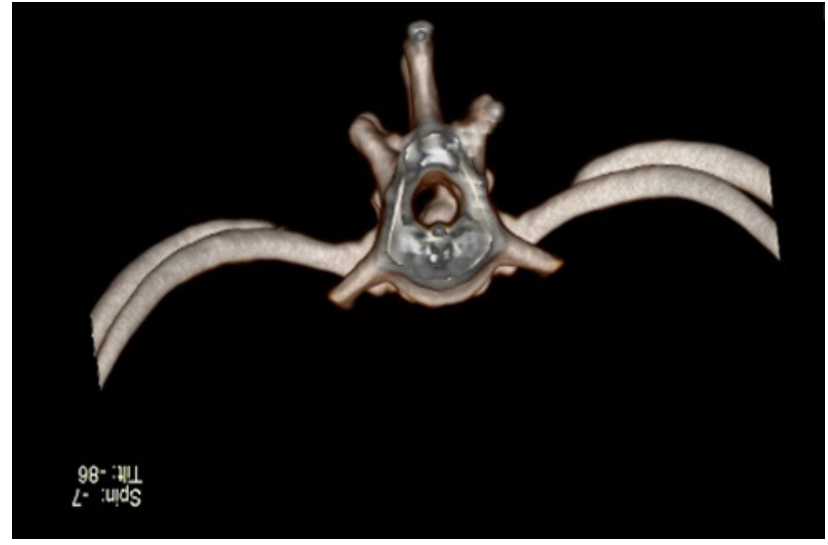

Figure 4. 3D rendering of lateral discal protrussion

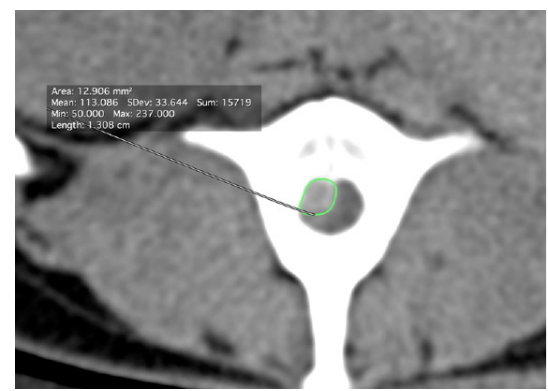

Figure 5. The hyperattenuating material closely to the protrusion site

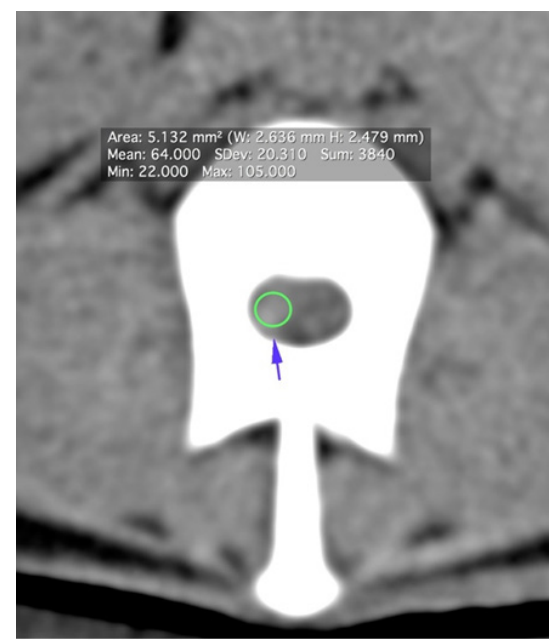

Figure 6. Hyper attenuated material, with displacement of the spine column

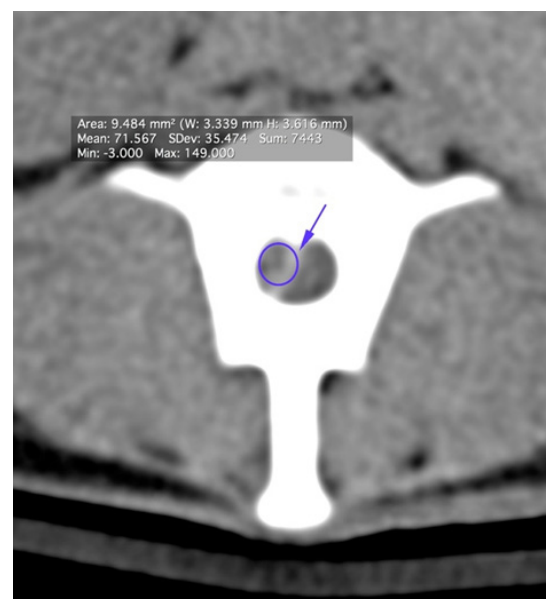

Figure 7. Post-contrast hyper attenuating lesion, the mean value of HU indicates a post compression hemorrhage.
I the A-index was $29.8 \pm 14.9 \%$, for Grade II $36.21 \pm 11.26 \%$, for Grade III $-44.53 \pm 12.31 \%$ and for Grade IV - 53.87 $\pm 21.23 \%$, values similarly with those obtained by Lim et al in 2010. The statistic doesn't show any significance between $\mathrm{H}$-index and the patient's grade.

The hyper attenuating material was measured closely to the protrusion area (figure 5) and compared with the normal HU value of the spinal cord.

In case of lack of calcified disc material, contrast substances were used to identify a possible hemorrhage or spinal inflammation (figure 6, figure 7).

In case of discospondilitis the CT reveal changes in the bone margins, and narrowing of the intervertebral space. Intervertebral disc end plates show irregularity and hyper intensification. Those changes indicate an inflammatory lesion of the end plates and the intervertebral disc that could be also associated with or without intervertebral disc protrusion (figure 8 , figure 9)

In case of inflammation there is a correlation between hyper intensification of the intervertebral endplates and presence of spondylitis. The normal HU value of the vertebrae and the endplates is $398.32 \pm 79.25 \mathrm{HU}$, in case of spondylitis the values reach $984.87 \pm 129.54 \mathrm{HU}$ (figure 10).

The presence of vacuum phenomena was rare being present in only two cases.

In case of hyperplastic lesions CT scans had revealed at the nerve roots an area of similar attenuation as spine and tissue directed right laterally to the spine and occupying the intervertebral canal in width of $7.57 \mathrm{~mm}$ (figure 11).

Other changes shown by CT scans revealed a mass of same attenuation as the surrounding tissue in the thorax region invading vertebral canal and vertebral bodies of T8-T10, with obvious 


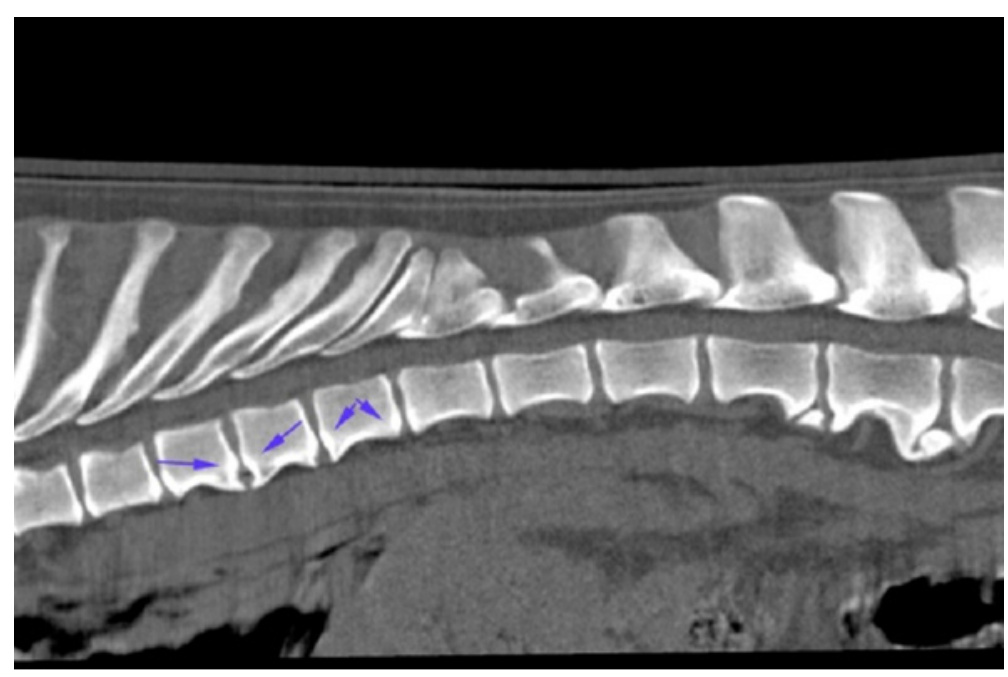

Figure 8. Hyperattenuating disc end plate

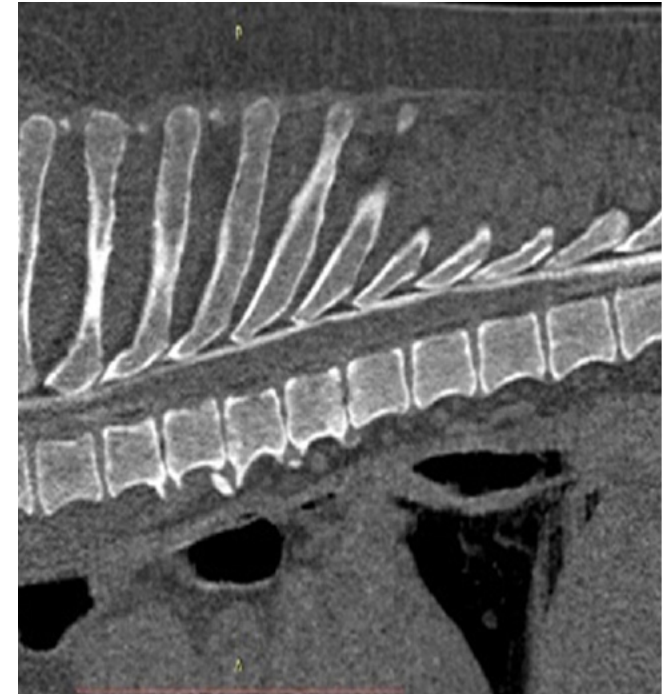

Figure 9. Post myelografy aspect of the spondilitis

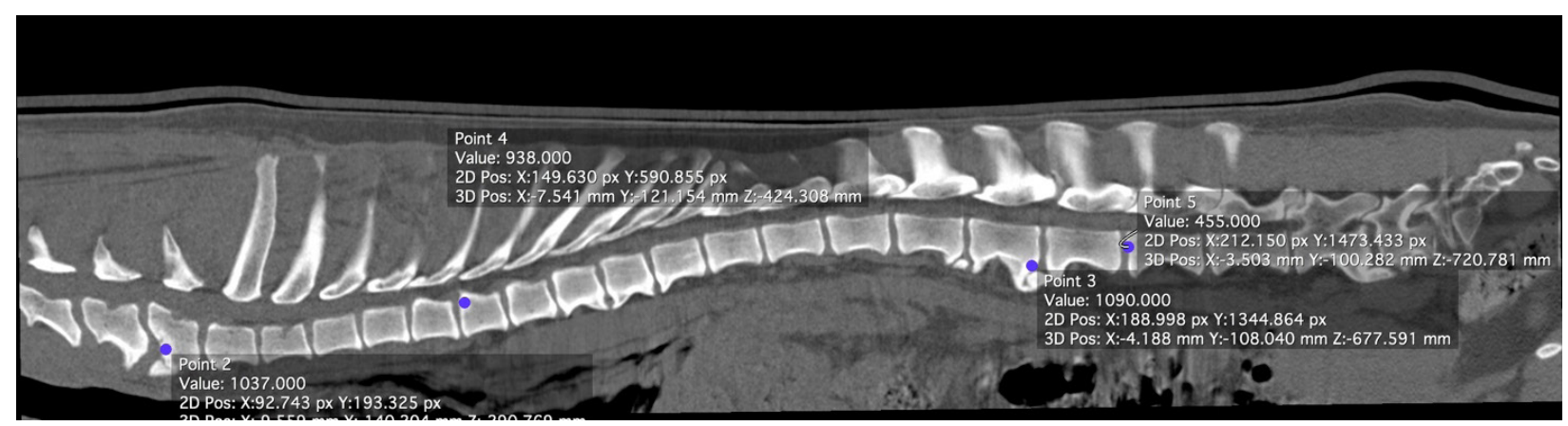

Figure 10. Hyperintensification of the vertebral endplates in spondylosis

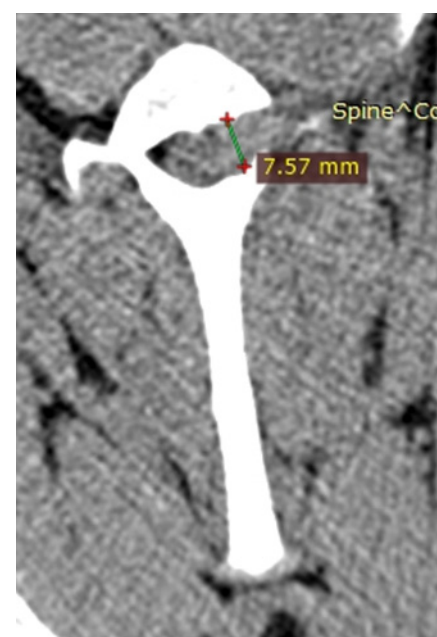

Figure 11. CT scan from amstaf,1y old, C6-7, intervertebral canal width measurement.

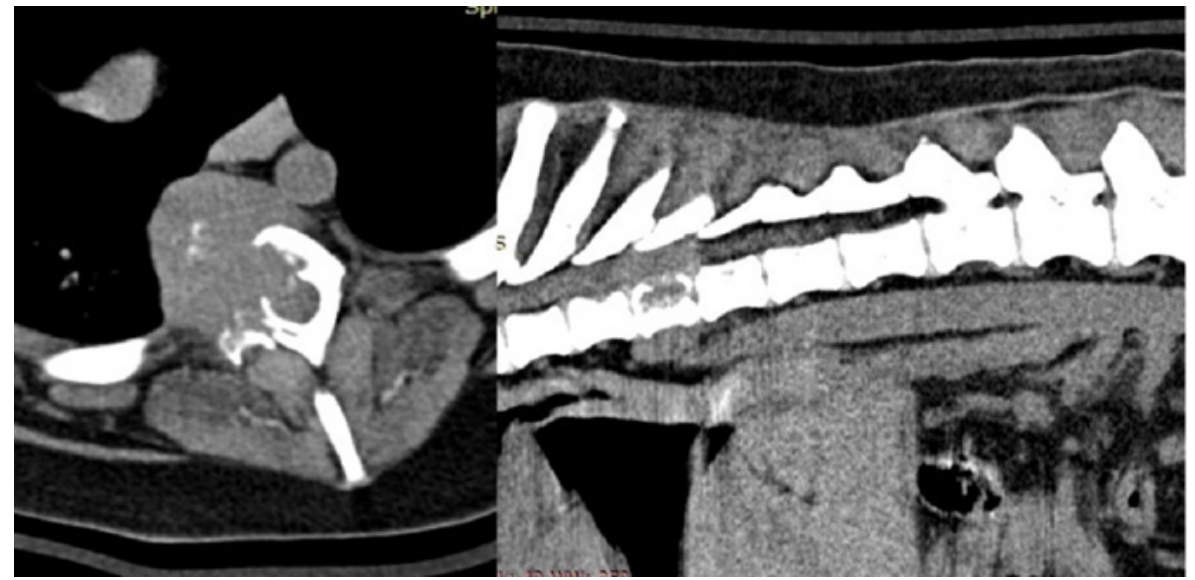

Figure 12. CT scans of the spine from golden retriever, 3y old, male, with destruction of vertebral body at the level of T8-T10, transvers image on the right, sagittal on the left destruction of the normal architecture of the spine, the mass oriented laterally left and was mixed in nature of type of tissue destroyed (figure 12).

\section{Conclusion}

The normal spinal cord, surrounded by epidural fat, is clearly visible dorsal to the inter- 
vertebral discs on transverse CT images. The outline of the spinal cord is less distinct dorsal to the vertebral bodies because of the relative absence of epidural fat, but the spinal cord is homogeneous in its attenuation characteristics. The intervertebral disc can be evaluated on the transverse CT images and mineralization of the nucleus pulposus is easily identified.

Normally, there is a mid-sagittal ridge of bone on the dorsal aspect of the vertebral body. In some dogs, there is also a small focus of mineralized material on the floor of the canal that is separate from the bone of the vertebra, in trying to identify this structure, it was noted that two dogs had a focal area with soft tissue attenuation characteristics in the same position as the mineralized focus, we therefore speculate that this focus could represent mineralization of the dorsal longitudinal ligament. This ligament lies on the floor of the ventral canal and fans out to a thin sheet over the intervertebral disc space, but narrows to a midline structure that is circular in cross section, dorsal to the vertebral body. This can potentially be mistaken for disc material, but can be identified as mineralized ligament by its position, its homogeneous hyper attenuating appearance and smooth borders (Lim et al., 2010).

The degenerative disc lesions were located in the T12-L1 area (38\%) and a percent of 34\% of the lesions were located in the L2-L7 area. In two cases the disc extrusion were located at the level of C2-C3, the presence of disc protrusion/extrusion are rare in the T1-T10 area (Sharp and Wheeler, 2005). The presence of the intercapital ligament enforce the ventral band that lie ventral to the dorsal ligament and join the ribs end between T2T11, helping resist the disc protrusion (Widmer and Thrall, 2002).

The hyper attenuated material surrounding the protrusion/extrusion of the intervertebral disc indicate calcification of the tissue if the attenuation is higher than $90 \mathrm{HU}$, if the attenuated material is between $60-90 \mathrm{HU}$ could easily indicate a hemorrhage (Kelender, 2005; Lim et al., 2010).

In case of endplate inflammation hyper intensification of the area was identify. CT is also useful to identify the changes in spine anatomy and bone structure.

CT diagnostic of spinal lesion help also the planning of the next intervention, recent studies show that preoperative planning based on MRI versus CT for spine compression influence the place and size of hemi laminectomy, with a tendency to approach a larger area in cases when the preoperative planning was done on MRI (Noyes et al., 2017). This aspect can influence the recovery time and also the vertebral column stability.

Base on the cases in our study, the CT have $94.48 \%$ sensitivity, $87.88 \%$ specificity, $97.47 \%$ positive predictive value and $76.32 \%$ negative predictive value in diagnostic of compressive spine lesions. Further studies are required to investigate the situation when non-calcified material or hemorrhages are presented at the protrusion/extrusion site and if administration of I.V. contrast agent can identify accurately those changes. Other direction of study of this subject should be CT evaluation in case of discospondilitis and metaplastic lesion of the spine.

Acknowledgement: This study was performed in the Radiology department of FMV Cluj Napoca being part of internal department research grant.

\section{References}

1. Chambers JN, Selcer BA, Butler TW, Oliver JE, Brown J (1994). A comparison of computed tomography and epidurography for the diagnosis of suspected compressive lesions at the lumbosacral junction in dogs. Prog Vet Neurol, 5: 30-34.

2. D'Anastasi M, Birkenmaier C, Schmidt GP, Wegener B, Reiser MF, Baur-Melnyk A (2011). Correlation between vacuum phenomenon on CT and fluid on MRI in degenerative disks. AJR Am J Roentgenol, 197: 1182-1189.

3. Drost WT, Love NE, Berry CR (1996). Comparison of radiography, myelography and computed tomography for the evaluation of canine vertebral and spinal cord tumors in sixteen dogs. Vet Rad Ultrasound, 37: 28- 33.

4. Feng SW, Chang MC, Wu HT, Yu JK, Wang ST, Liu CL (2011). Are intravertebral vacuum phenomena benign lesions? Eur Spine J, 20: 1341-1348.

5. Freeman AC, Kent M, Platt SR (2010). Intervertebral disc disease. In: R.V. Morgan (Ed.), Small Animal Practice Client Handouts (pp. 116-117). Philadelphia: Saunders.

6. Hoerlein BF (1971). Canine Neurology: Diagnosis and Treatment (2nd ed.). Philadelphia: Saunders (pp. 307391).

7. Kalender WA (2005). Computed Tomography Fundamentals, System Technology, Image Quality, Applications (2nd ed.). Erlangen: Publicis Corporate Publishing (pp. 17-97).

8. Kirberger RM, Roos CJ, Lubbe AM (1992). The radiological diagnosis of thoracolumbar disc disease in the Dachshund. Vet Radiol Ultrasound, 33: 255-261.

9. Knutson F (1942). The vacuum phenomenon in the intervertebral disks. Acta Radiol, 4: 173-179. 
10. Lamb CR (1994). Common difficulties with myelographic diagnosis of acute intervertebral disc prolapse in the dog. J Small Anim Pract, 35: 549-558.

11. Lim C, Kweon OK, Choi MC, Choi J, Yoon J (2010). Computed tomographic characteristics of acute thoracolumbar intervertebral disc disease in dogs. J Vet Sci, 11: 73-79.

12. Liptak JM, Watt PR, Thomson MJ, Copeland SE, Galloway AM (1999). Hansen type I disk disease at T1-2 in a dachshund. Aust Vet J, 77: 156-159.

13. Noyes JA, Thomovsky SA, Chen A, Owen TJ, Fransson BA, Carbonneau KJ, Matthew SM (2017). Magnetic resonance imaging versus computed tomography to plan hemilaminectomies in chondrodystrophic dogs with intervertebral disc extrusion. Veterinary Surgery, 1-7.

14. Ohlerth S, Scharf G (2007). Computed tomography in small animals - Basic principles and state of the art applications. The Veterinary Journal, 173: 254-271.

15. Olby NJ, Muñana KR, Sharp NJH, Thrall DE (2000). The computed tomographic appearance of acute thoracolumbar intervertebral disc herniations in dogs. Vet Radiol Ultrasound, 41: 396-402.

16. Sharp NJH, Wheeler SJ (2005). Small Animal Spinal Disorders: Diagnosis and Surgery (2nd ed.). Edinburgh: Elsevier (pp. 1-17, 121-135).

17. Sra P, Aldrich EM, Bernbeck JA, Delamarter RB (1999). Evaluation of locomotor rating scales following spinal cord injury in rats, Anaheim, California 45th Annual Meeting, Orthopaedic Research Society, pp. 982.

18. Turrel JM, Fike JR, LeCouteur R, Higgins RJ (1986). Computed tomographic characteristics of primary brain tumors in 50 dogs. J Am Vet Med Assoc, 188: 851-856.

19. Widmer WR, Thrall DE (2002). Canine and feline intervertebral disc disease, myelography, and spinal cord disease. In: D. E. Thrall (ed.). Textbook of Veterinary Diagnostic Radiology (4th ed.) (pp. 110-133). Philadelphia: Saunders. 